\title{
Distance and Size of the Red Hypergiant NML Cyg
}

\author{
B. Zhang ${ }^{1,2}$, M. J. Reid ${ }^{3}$, K. M. Menten ${ }^{1}$, X. W. Zheng ${ }^{4}$ \\ and A. Brunthaler ${ }^{1}$ \\ ${ }^{1}$ Max-Plank-Institut für Radioastronomie, Auf dem Hügel 69, 53121 Bonn, Germany \\ email:bzhang@mpifr.de \\ ${ }^{2}$ Shanghai Astronomical Observatory, Chinese Academy of Sciences, 80 Nandan Road, \\ Shanghai 200030, China \\ ${ }^{3}$ Harvard-Smithsonian Center for Astrophysics, 60 Garden Street, Cambridge, MA 02138, USA \\ ${ }^{4}$ Department of Astronomy, Nanjing University, 22 Hankou Road, Nanjing 210093, China
}

\begin{abstract}
We present astrometric results of phase-referencing VLBI observations of $22 \mathrm{GHz}$ $\mathrm{H}_{2} \mathrm{O}$ maser and $43 \mathrm{GHz} \mathrm{SiO}$ maser emission towards the red hypergiant NML Cyg using VLBA. We obtained an annual parallax of $0.62 \pm 0.04$ mas, corresponding to a distance of $1.61_{-0.11}^{+0.13}$ kpc. With a VLA observation in its largest (A) configuration at $43 \mathrm{GHz}$, we barely resolve the radio photosphere of NML Cyg, and find a uniform-disk diameter of $44 \pm 16$ mas.
\end{abstract}

Keywords. astrometry — masers — instrumentation: interferometric — stars: distance stars: individual (NML Cyg) — supergiants

\section{Introduction}

NML Cyg has been assumed to be at the same distance as the Cyg OB2 association (Morris \& Jura 1983). The luminosity of NML Cyg derived using the previous estimated distance of $1.74 \pm 0.2 \mathrm{kpc}$ by Massey \& Thompson (1991) places it near the empirical upper luminosity boundary on the H-R diagram (Schuster et al. 2006). However, a luminosity estimate of a star is dependent on the square of its distance. Thus, a trustworthy distance is crucial to derive a reliable luminosity of NML Cyg. In addition to the distance, another fundamental stellar parameter is the size. NML Cyg's high massloss rate results in a dense circumstellar envelope, thus the star is hardly observable at visual wavelengths due to high extinction, while it is extremely luminous in the infrared. Blöcker et al. (2001) obtained a central star luminosity by integrating the spectral energy distribution from 2 to $50 \mu \mathrm{m}$, and derived a stellar diameter of 16.2 mas assuming $T_{\text {eff }}=$ $2500 \mathrm{~K}$. However, a direct detection of the star would be superior to indirect methods. Radio continuum emission from the evolved star's photosphere can be imaged by VLA using $\mathrm{SiO}$ masers as a phase reference (Reid \& Menten 2007; Zhang et al. 2012), this allows us to directly determine the size of NML Cyg's radio photosphere.

\section{Parallax of NML Cyg}

We conducted VLBI phase-referencing observations of circumstellar $22 \mathrm{GHz} \mathrm{H}_{2} \mathrm{O}$ and $43 \mathrm{GHz} \mathrm{SiO}$ masers toward NML Cyg and several extragalactic radio sources with the VLBA at five epochs in one year. We identified about $20 \mathrm{H}_{2} \mathrm{O}$ maser features with a $V_{\mathrm{LSR}}$ range of $-25.4-6 \mathrm{~km} \mathrm{~s}^{-1}$, while the $\mathrm{SiO}$ masers were very weak during our observation and only detectable at the last epoch. Using the positions of $18 \mathrm{H}_{2} \mathrm{O}$ maser spots relative to three background sources J2044+4005, J2046+4106 and J2049+4118 within $2^{\circ}$ of 

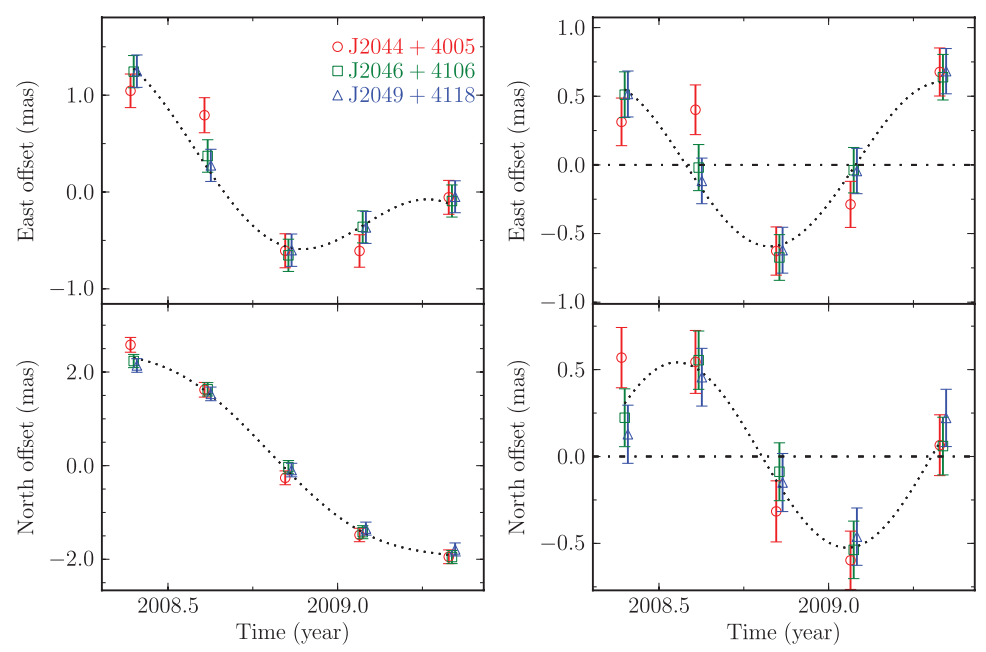

Figure 1. Parallax and proper motion data (markers) and best-fitting models (dotted lines) for the maser spot at the $V_{\mathrm{LSR}}$ of $6.48 \mathrm{~km} \mathrm{~s}^{-1}$. Plotted are positions of the maser spot relative to the extragalactic radio sources J2044+4005 (circles), J2046+4106 (squares) and J2049+4118 (triangles). Left panel: Eastward (solid lines) and northward (dotted lines) offsets and best-fitting models versus time. Data of the eastward (upper panel) and northward (bottom panel) positions are offset horizontally for clarity. Right panel: Same as the left panel, except the best-fitting proper motion has been removed, displaying only the parallax signature.

NML Cyg, we determined a trigonometric parallax of $0.62 \pm 0.04$ mas, corresponding to a distance of $1.61_{-0.11}^{+0.13} \mathrm{kpc}$. Fig. 1 shows the parallax fit of one of the maser spots as an example.

\section{Radio Photosphere of NML Cyg}

We detected weak continuum emission at $43 \mathrm{GHz}$ from the radio photosphere of NML Cyg using a calibration scheme in which circumstellar masers provide a phase reference for the continuum data with VLA. Taking into consideration possible biases introduced by the deconvolution process of images at low SNR, we fitted a round disk model to the $u v$-data directly and obtained a diameter of $44 \pm 16$ mas, which is comparable with two times that derived from the Stefan-Boltzmann law with a revised luminosity according to our distance and $T_{\text {eff }}=2500 \mathrm{~K}$.

\section{Acknowledgements}

B. Zhang is supported by the National Science Foundation of China under grant 11073046, 11133008 .

\section{References}

Blöcker, T., Balega, Y., Hofmann, K.-H., \& Weigelt, G. 2001, Aש\&A, 369, 142

Massey, P. \& Thompson, A. B. 1991, AJ, 101, 1408

Morris, M. \& Jura, M. 1983, ApJ, 267, 179

Reid, M. J. \& Menten, K. M. 2007, ApJ, 671, 2068

Schuster, M. T., Humphreys, R. M., \& Marengo, M. 2006, AJ, 131, 603

Schuster, M. T., et al. 2009, ApJ, 699, 1423

Zhang, B., Reid, M. J., Menten, K. M., \& Zheng, X. W. 2012, ApJ, 744, 23 


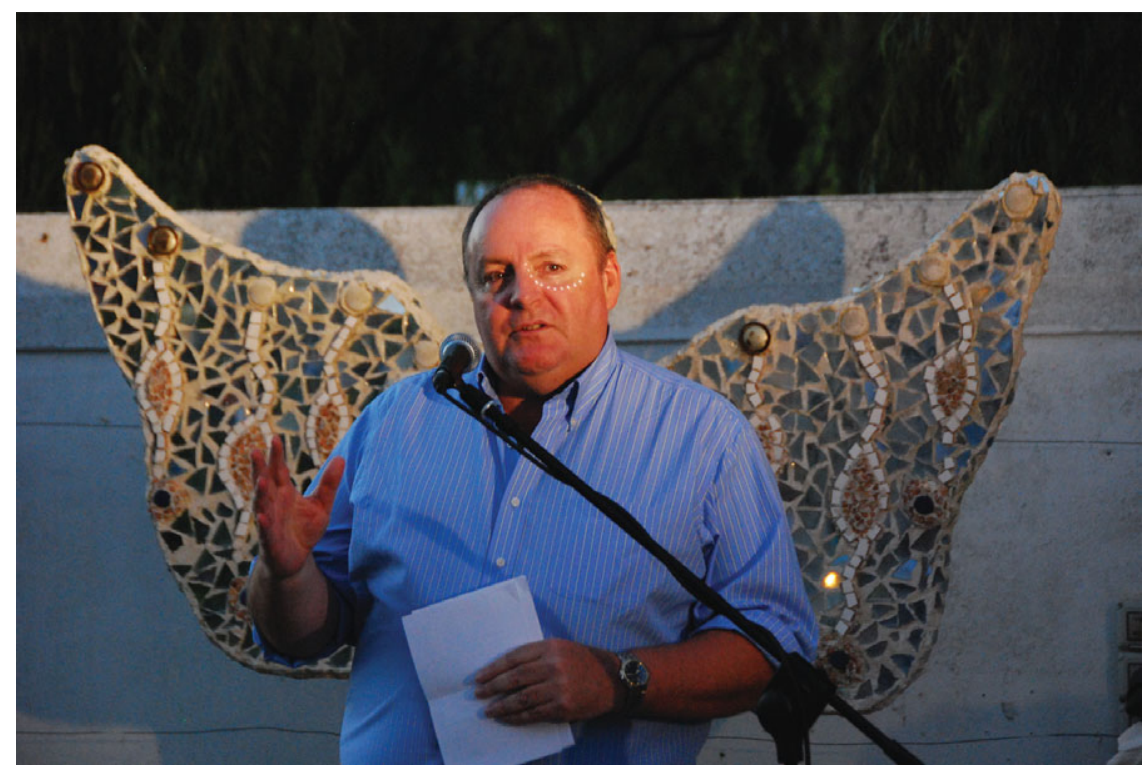

A memorable before dinner speech by Phil Diamond. 